\title{
Experimental Investigation of Packed Bed Latent Thermal Energy Storage
}

\author{
Shubham Sahu, Dinesh Kumar Sharma, Manu Augustine, Sanjay Choudhary \\ Department of Mechanical Engineering, Swami Keshvanand Institute of Technology, Management \& \\ Gramothan, Jaipur -302017 (INDIA) \\ Email: sahu20shubham@gmail.com, Dinesh.sharma@skit.ac.in, augustine.manu@gmail.com, \\ sanjay.sahu.mech@gmail.com \\ Received 18.12.2020, received in revised form 23.12.2020, accepted 24.12.2020
}

\begin{abstract}
Renewable energy resources such as solar energy are essential to play an important role to increase the energy production share. However, solar energy is available for limited hours and that also having variations in its intensity. Therefore, thermal energy storage is essential to accommodate within solar thermal systems for consistency in operation. High energy storage density and proper heat transfer are essential requirements from thermal energy storage. Latent thermal energy storage (LTES) is a preferred choice for storing thermal energy in a compact space compared to sensible thermal energy storage (STES). From the previous studies, it is reported that previously designed LTES with heat exchangers have not proven effective due to improper heat transfer because of the low heat transfer area and complicated designs. Hence, the packed bed thermal energy concept is discussed in the literature to carry out proper heat transfer and simple design.

In this presented study, a packed bed latent thermal energy storage is developed by placing PCM (Paraffin wax $58-60^{\circ} \mathrm{C}$ ) filled within spherical shaped balls of HDPE material inside a TES of cylindrical shape. Charging and discharging loops are actualized with the help of heater and radiator with exhaust fan respectively. An experimental investigation is carried out for the analysis of the charging and discharging behaviour of the developed PBLTES. A total of 9 modes are analyzed for analyzing the charging/discharging behaviour of PBLTES developed
\end{abstract}

Keywords- Latent Thermal Energy Storage; Encapsulation; Phase Change Material

\section{INTRODUCTION}

Energy is the backbone of the economic prosperity of any nation. Energy demands are increasing in every sector day by day. There are limited sources of conventional fuels such as coal, petroleum, nuclear, etc. which are depleting very fast over the past few decades. Renewable energy solution such as solar energy seems to be effective being abundant, non-polluting, and economical. Since solar energy is only available for a limited period around the day and that also with varying intensity. Therefore, a type of thermal energy storage (TES) is always recommended for the proper utilization of solar thermal energy. A TES helps to overcome the discrepancy between the thermal energy transactions and also stores thermal energy for later use. A TES system can store all types of thermal energy available i.e. solar energy, surplus thermal energy, waste heat from plants, industries, etc. Different types of TES system include sensible thermal energy storage (STES), latent thermal energy storage (LTES), and thermochemical energy storage (TCES). STES is one of the commonly used TES because of its ease of development and operation but possess low energy density. While, LTES offers high energy storage density along with nearly isothermal charging/discharging utilizing latent heat of phase transition of phase change materials (PCMs). LTES reported designs are mostly observing the issue of improper energy storage and release due to ineffective heat transfer mechanisms. An LTES typically requires well-designed heat exchangers to charge/discharge the system. This restricted the development of the LTES for its implementation on real-size projects.

PCM integrated TES system is widely used in numerous applications such as thermal storage in buildings, storage of solar thermal energy, domestic water, engine cooling, space heating, etc. Thermal energy store/release in this is processed when PCM is exposed to hot/cold surroundings, it undergoes melting (charging process) and solidification (discharging process) respectively for hot storage and vice-versa for the cold storage [1]. However, the LTES suffers from the low thermal conductivity of the PCMs. This restricts the charging and discharging rates of the TES. Different thermal conductivity enhancement techniques are proposed to overcome this issue such as mixing of high thermal conductivity particles into PCM, encapsulation of PCMs, metal matrix, graphite matrix, etc. [2-3].

Encapsulation of PCMs offers a large heat transfer area to the PCM expanding the thermal conductivity of the inactive heat thermal capacity framework. This process was first invented by Barrett $\mathrm{K}$ green in the 1950s and 1960s [4]. Encapsulation separates the PCM from its surrounding and offers large heat transfer area at the same time. Some PCMs come in the category of hazardous, encapsulation helps to contain these and allowing its use for applications such as food storage, blood transport, building cooling/ heating, etc[5]. 
Skar et al. [6] encapsulated PCM in different configurations (cuboids, cone, sphere, pyramid and cylinder) and reported freezing/melting through experimental investigations performed. Water was used as a PCM. It was concluded on the basis of reported investigation that spherical capsules performed effective energy storage/release compared to other configurations. It was also identified that packing of capsules played an important role. Specially packed encapsulated LTES performed better compared to random packing.

ElGhnam et al. [8] performed charging and discharging of water (PCM) filled inside spherical capsules for the purpose of building airconditioning. The effect of encapsulation materials and diameter of spherical capsules were reported. The aqueous solution of $35-\mathrm{wt} \%$ ethylene glycol was used as the heat transfer fluid. Smaller diameter capsules showed improved heat transfer characteristics whereas HTF temperature was effective as higher. Whereas discharging rate was decreased for above configuration. Capsules of metals showed better heat transfer compared to nonmetals but difference was not significant. Thus it was noted that capsule material is of less dominant over other geometrical and operational parameters tested.

In the presented work, a packed bed latent thermal energy storage (PBLTES) is developed, and further, an experimental investigation is carried out to analyze the charging and discharging behavior under various modes of operations keeping different HTF temperatures and volume flow rates.

Table 1 Modes of the experimental plan

\begin{tabular}{|l|c|c|c|}
\hline Mass flow rate & \multicolumn{3}{|c|}{ Cut off temperature } \\
\hline 7 LPM (charging and discharging) & $65^{\circ} \mathrm{C}$ & $70^{\circ} \mathrm{C}$ & $75^{\circ} \mathrm{C}$ \\
\hline 8 LPM (charging and discharging) & $65^{\circ} \mathrm{C}$ & $70^{\circ} \mathrm{C}$ & $75^{\circ} \mathrm{C}$ \\
\hline 9 LPM (charging and discharging) & $65^{\circ} \mathrm{C}$ & $70^{\circ} \mathrm{C}$ & $75^{\circ} \mathrm{C}$ \\
\hline
\end{tabular}

\section{DESCRIPTION OF THE EXPERIMENTAL SETUP}

The experimental setup consists of a PBTES designed with a PCM (Paraffin wax $58-60^{\circ} \mathrm{C}$ ) contained in HDPE spherical balls and arranged systematically inside a cylindrical TES. A total number of 85 balls are arranged in an order of 5 balls per row and a total of 17 rows. Additionally, centrifugal pump, heater, and radiator equipped with an exhaust fan are also managed for charging and discharging loops demonstration of for PBLTES. The flow meter and thermocouples are used for flow and temperature measurements at appropriate locations to record the behavior of the developed system. A data logger is also used to integrate different results into one single file that further helped to process the data.

\subsection{Mode 1: Volume Flow of 9 LPM and Heater Cut- Off at $65^{\circ} \mathrm{C}(\mathrm{V} 9 \mathrm{CO} 55)$}

In Mode 1, the volume flow rate is further increased keeping the heater cut-off closer to $65^{\circ} \mathrm{C}$. Figure 3 reports the variation of temperatures at the inlet and outlet section of the PBLTES. These variations reflect the charging and discharging of the PCM filled inside the spherical capsules. With an increase in the volume flow rate, charging time has been increased considerably and so the discharging time compared to Mode 1 and Mode 4.

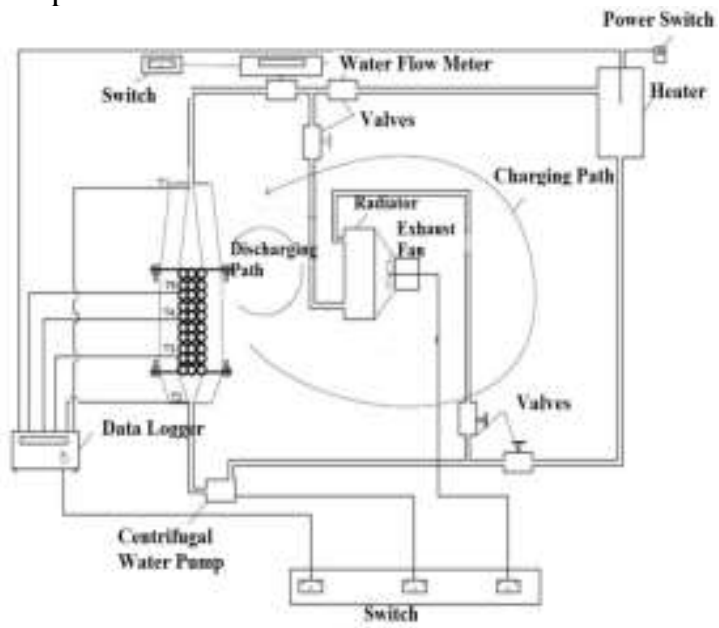

Fig.1 Schematic diagram of the experiment setup

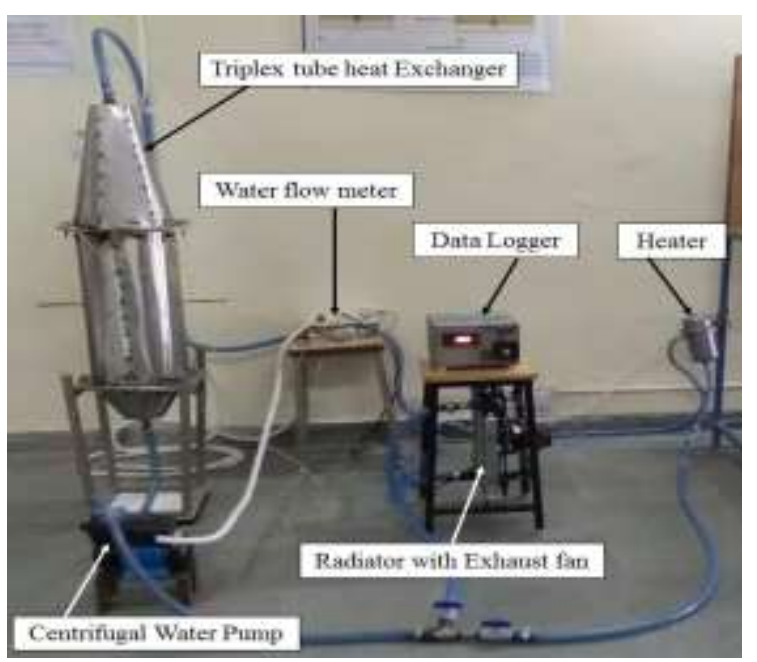

Fig.2 Actual experimental setup

Table 2 Components and their capacity

\begin{tabular}{|c|c|}
\hline Component & Capacity/ Power / Type \\
\hline $\begin{array}{l}\text { Thermal Energy } \\
\text { Storage Tank }\end{array}$ & The volume of cylinder- $0.0257 \mathrm{~m}^{3}$ \\
\hline $\begin{array}{l}\text { Centrifugal Water } \\
\text { Pump }\end{array}$ & Power input-0.5 HP/.37 kW \\
\hline Water Flow Meter & Mechanical flow meter \\
\hline Thermocouples & K-type \\
\hline Radiator & Surface area- $7284.73 \mathrm{~mm}^{2}$ Seamless \\
\hline Exhaust Fan & R.P.M.- 2800, Air delivery- $2120 \mathrm{~m}^{3} / \mathrm{h}$ \\
\hline Heater & Power $-3000 \mathrm{~W}$ \\
\hline Data Logger & 6 channel data logger \\
\hline PCM & Latent heat of fusion- $214 \mathrm{~kJ} / \mathrm{kg}$ \\
\hline Spherical balls & $\begin{array}{c}\text { High-density polyethylene, diameter- } \\
0.034 \mathrm{~m}\end{array}$ \\
\hline
\end{tabular}




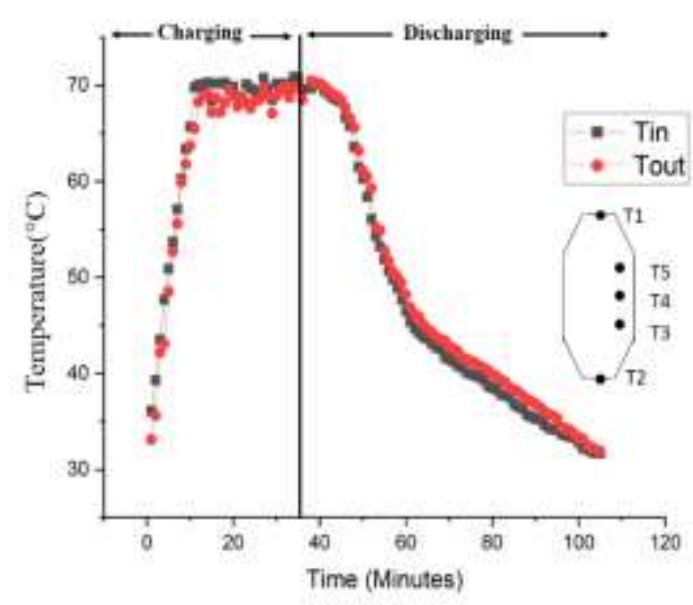

Fig.3 Variation of Temperatures $T_{1}$ and $T_{2}$ concerning time while charging and discharging at Mode 1

Figure 4 reports the variation of temperatures inside the spherical capsules where PCM is filled. It can be seen that during the charging T5, which is placed on top is having the highest temperature change when compared with the other two.

It may be because hot HTF first comes in contact with the upper part of TES. While during discharging, top layers are observing the opposite trends. T3 is placed bottom of the storage so the lowest temperature occurs because HTF comes in contact at the end during charging and the highest temperature during discharging.T4 always having a middle temperature during charging and discharging. This shows the thermal stratification zone inside the PBLTES and also reflects the proper heat transfer within.

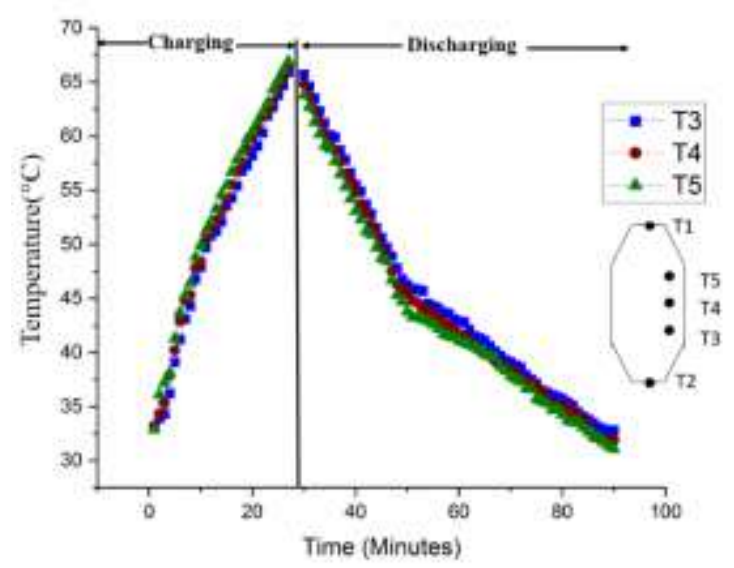

Fig.4 Variation of Temperatures $\mathrm{T}_{3}, \mathrm{~T}_{4}$, and $\mathrm{T}_{5}$ of PCM inside TES while charging and discharging at Mode 1

\subsection{Mode 2: Volume Flow of 9 LPM and Heater Cut- Off at $70^{\circ} \mathrm{C}(\mathrm{V} 9 \mathrm{CO} 0)$}

Figure 5 is helpful to note the thermal energy distribution inside TES. PCM spherical capsules have been charged and discharged as expected. Similar to the previous case, the upper part of the TES has the highest temperature during charging as it comes in the first contact and the lowest temperature during discharge.

Again, during charging, the bottom of the cylinder has the lowest temperature and the highest temperature during discharge.

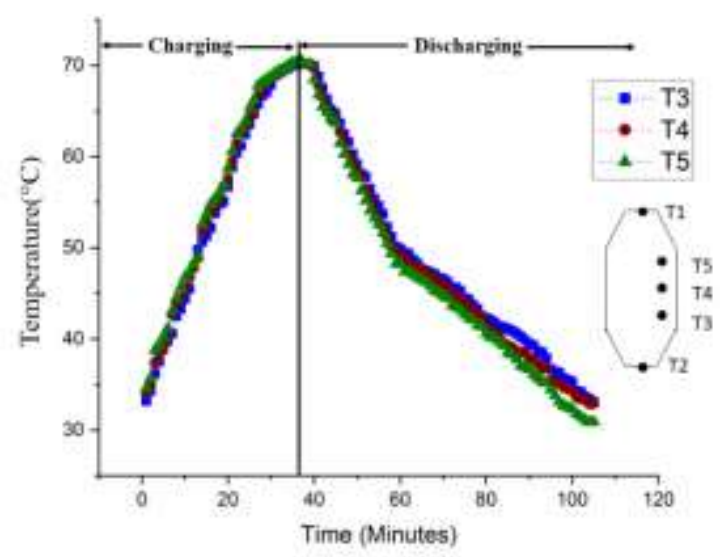

Fig.5 Variation of Temperatures T3, T4, and T5 of PCM inside TES while charging and discharging at Mode 2

\subsection{Mode 3: Volume Flow of 9 LPM and Heater Cut- Off at $75^{\circ} \mathrm{C}$ ( $\mathrm{V} 9 \mathrm{CO}$ - 5$)$}

Figure 6 shows the variation of temperatures from the thermocouples placed inside the LHTES. These behaviors are equivalent to the charging and discharging of the previous modes. The upper part of the cylinder has the highest temperature during charging since it comes into contact first, and the lowest temperature during discharge, similar to the previous case. Again, the lower portion of the cylinder has the lowest temperature during charging and the maximum temperature during discharge.

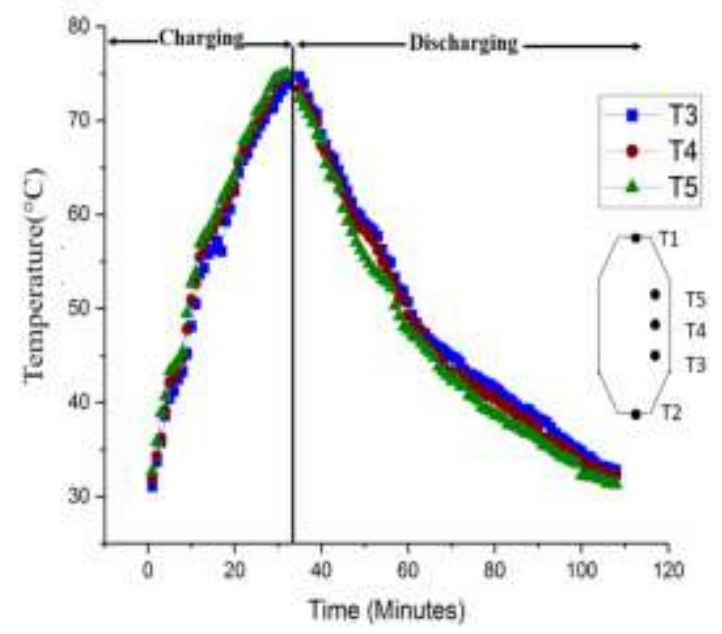

Fig.6 Variation of Temperatures T3, T4, and T5 of PCM inside TES while charging and discharging at Mode 3

\subsection{Comparative Analysis of Energy Stored within} PCM

Figure 7 shows all volume flow rate and cut-off temperature. It can be observed from this figure that a shorter charging time and higher amount of energy stored is for the cases with high heater cut-off temperature and lower volume flow rates (i.e. 
QPCM 7-75 ${ }^{\circ}$ ). The lowest volume flow rate at the higher heater cut-off temperature has the highest stored energy and also has the shortest charging time. This is mainly because of the high-temperature difference between PCM and HTF, which allows a high heat transfer rate compared to the other two.

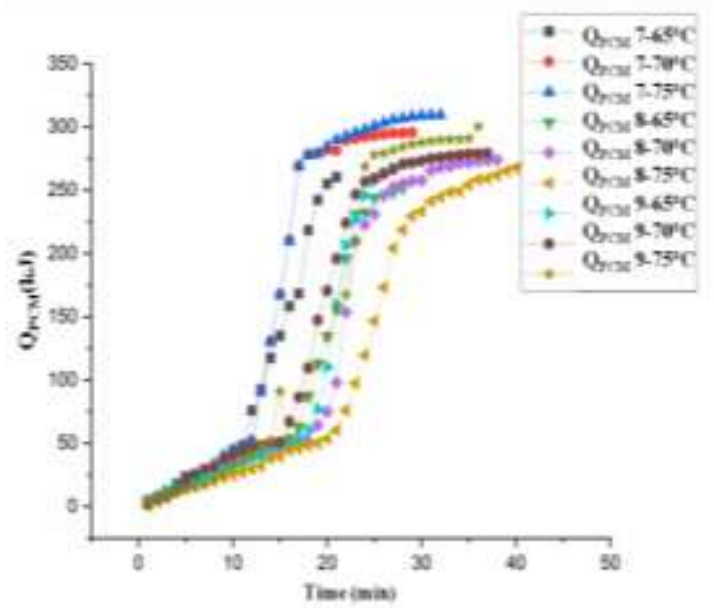

Fig.7 Variation of energy stored for all volume flow rate and all cut-off temperature

\subsection{Comparative Analysis of Melting Fraction}

Figure 8 shows the effect of volume flow rates and heater cut-off temperature on the melting fraction of PCM. It is noted that for the same volume flow rate higher temperature has a lower melting fraction and the lower temperature has a higher melting fraction. At any instant, a lower temperature has a higher melting fraction. Concerning the time lower temperature takes more time compared to a higher temperature.

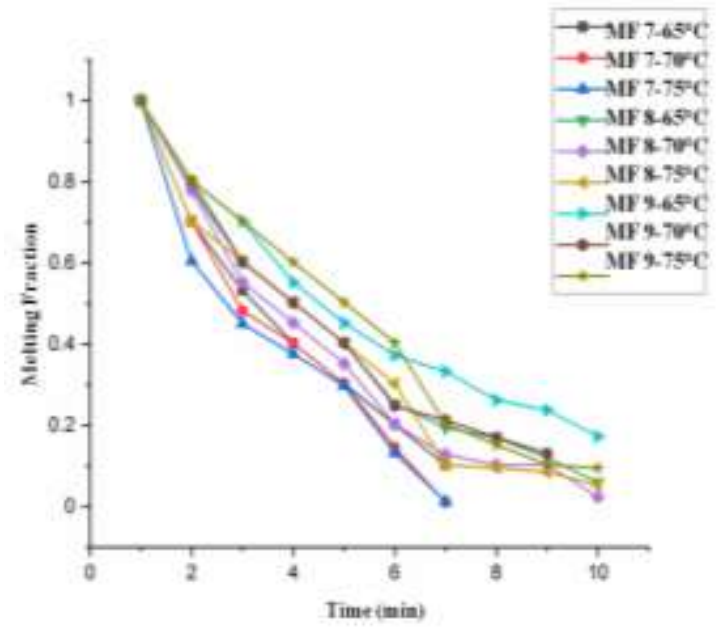

Fig.8 Variation of melting fraction for all volume flow rate and all cut-off temperature

\section{CONCLUSION}

In the present work, the charging and discharging behavior of developed PBLTES are reported for different operating conditions based upon the experimental investigations performed. The following conclusions are made based on the above results:

1. The temperature profiles in all modes indicated a proper heat transfer within PCM.

2. In all modes, while charging the PCM inside the PBLTES showed that lower portion tank (lower portion of balls cage) compared to the upper and middle portions.

3. In all modes, while discharging the PCM inside the LHTES showed that upper portion tank (lower portion of balls cage) compared to the lower and middle portions.

4. Based on the overall comparison it has been reported the lower volume flow rate and higher heater cut-off temperature shows the effective heat transfer within the developed Packed Bed Latent Thermal Energy Storage.

Therefore, it is concluded that the use of encapsulated PCM in TES helps to overcome the issue of improper heat transfer through latent thermal energy storage as compared to those with heat exchangers. Also, it is evident from this experimental investigation that PBLTES shows high energy storage density compared to conventional sensible thermal energy storage. Thus, it can be clearly understood that the concept of high energy storage density for a narrow temperature range is the feature of latent thermal energy storage.

\section{REFERENCES}

[1] Sharma SomeshowerDutt, Kitano Hiroaki, and SagaraKazunobu, "Phase Change Materials for LowTemperature Solar Thermal Applications', Res. Rep. Fac. Eng. Mie Univ., Vol. 29, pp. 31-64, 2004

[2] Tan, F.L., Hosseinizadeh, S.F., Khodadadi, J.M., and Fan, L., 2009. Experimental and computational study of constrained melting of phase change materials (PCM) inside a spherical capsule. International Journal of Heat and Mass Transfer, 52(15-16), pp.3464-3472.

[3] Cheralathan, M., Velraj, R., and Renganarayanan, S., 2006 Heat transfer and parametric studies of an encapsulated phase change material based cool thermal energy storage system. Journal of Zhejiang University-SCIENCE A, 7(11), pp.1886-1895.

[4] Amin, N.A.M., Bruno, F., and Belusko, M., 2014. Effective thermal conductivity for melting in PCM encapsulated in a sphere. Applied Energy, 122, pp.280-287.

[5] Hawlader, M.N.A., Uddin, M.S., and Khin, M.M., 2003. Microencapsulated PCM thermal-energy storage system. Applied Energy, 74(1-2), pp.195-202.

[6] ElGhnam, R.I., Abdelaziz, R.A., Sakr, M.H. and Abdelrhman, H.E., 2012. An experimental study of freezing and melting of water inside spherical capsules used in thermal energy storage systems. Ain Shams Engineering Journal, 3(1), pp.33-48.

[7] Susskind, H., and Becker, W., 1967. Pressure drop in geometrically ordered packed beds of spheres. AIChE Journal, 13(6), pp.1155-1159.

[8] ElGhnam, R.I., Abdelaziz, R.A., Sakr, M.H. and Abdelrhman, H.E., 2012. An experimental study of freezing and melting of water inside spherical capsules used in thermal energy storage systems. Ain Shams Engineering Journal, 3(1), pp.33-48. 